\title{
How to Recover Causality for Tachyons Even in Macrophysics.
}

\author{
M. Pavšič and E. ReCAMI \\ Istituto di Fisica Teorica dell'Università - Catania, Italia
}

(Nuovo Cimento, 36 A, 171 (1976))

In this paper we considered in particular a macro-object $B$, with rest-mass $M \equiv M_{B}$ and 3-momentum $\boldsymbol{P}$, moving with (subluminal) speed in the positive $x$-direction and absorbing tachyons, with rest-mass $m \equiv m_{0}$ and 3-momentum $p$, moving along the positive $x$-axis as well. From the four-momentum conservation law in the case when $M$ does not change during the absorption, we correctly derived eq. (2). In the case, however, when we have a nonzero $\Delta \equiv M^{\prime 2}-M^{2}$, then eq. ( 2 bis) was trivially miscalculated. It should rather read

(2 bis) $\quad 2 M^{2}|\boldsymbol{p}|=\left(m^{2}+\Delta\right)|\boldsymbol{P}|+E \sqrt{\left(m^{2}+\Delta\right)^{2}+4 m^{2} M^{2}}, \quad E \equiv \sqrt{\overline{\boldsymbol{P}^{2}+M^{2}}}$.

(Notice that the present $\Delta$ was called $\Delta^{2}$, since in this paper we assumed $\Delta>0$.)

Notice, however, that the physical conclusions-and actually the whole paper-remain totally unchanged. Even fig. 5 still holds, since the physics was not affected at all by that miscalculation.

Let us take advantage of the present occasion for repeating that--as already ( $\left.{ }^{1}\right)$ remarked--lines 14 and 15 (and the first eight words of line 16) ought to be eliminated at page 178 .

For further details about the subjects of this paper, see for instance ref. $\left({ }^{1,2}\right)$.

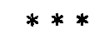

The authors are grateful to Prof. R. G. GLASser for the kind collaboration.

(1) E. Recami : Lett. Nuovo Cimento, 21, 208 (1978), and to appear.

(2) P. Caldirola and E. RECAMI: to appear in Boston Studies in the Philosophy of Sciences, edited by R. S. Cohen and M. Dalla Chiara (Dordrecht); E. Recami: Lett. Nuovo Cimento, 18, 501 (1977); E. RECAMI, Editor: Tachyons, Monopoles, and Related Topics (Amsterdam, 1978). See also V. De Sabbata, M. Pavšı̌ and E. Recami: Lett. Nuovo Cimento, 19, 441 (1977). 